\title{
Short-term memory for Chinese characters and radicals
}

\author{
CHIH-WEI HUE and JAMES R. ERICKSON \\ University of Texas at Arlington, Arlington, Texas
}

\begin{abstract}
Short-term memory for Chinese radicals and characters, varying in orthographic complexity, frequency, and-for radicals-intercharacter frequency (the number of compound characters that contain the radical), was studied using an immediate free-recall task. When radicals or characters are relatively frequent, so that their pronunciations are well known by literate Chinese, they seem to be maintained in verbal form in short-term memory. For these stimuli, intercharacter frequency and complexity have relatively small influences on memory span. Stimuli low in frequency, with pronunciations that are not apt to be known, seem to be maintained in visual form in short-term memory. Memory span is much smaller for these stimuli and is influenced by both intercharacter frequency and complexity. Furthermore, short-term memory for relatively highfrequency characters is interfered with more by a verbal than by a visual intervening task, whereas the opposite is true for low-frequency characters.
\end{abstract}

Baddeley and his colleagues (Baddeley, 1981, 1986; Baddeley, Grant, Wight, \& Thomson, 1975; Baddeley \& Lieberman, 1980) have proposed that there are at least two types of short-term memory: verbal and visual. Although the existence of a visual short-term memory has been supported for nonverbal stimuli (e.g., Baddeley \& Lieberman, 1980; Cermak, 1971), its involvement in memory for verbal stimuli has not been demonstrated conclusively (e.g., Baddeley, 1986; Frick, 1985; Laughery, Welte, \& Spector, 1973). The experiments reported here provide further information on visual and verbal shortterm memory, using nonalphabetic linguistic stimuli, specifically Chinese radicals and characters.

In contrast to the English alphabetic system, the Chinese written language is logographic. Each of the more than 10,000 Chinese characters in existence is made up of a combination of one or more of about 20 basic strokes. Although the definition of a stroke may vary from scholar to scholar, 8 of the most common are illustrated in Figure 1, along with a character meaning "eternity" constructed from these strokes.

A number of recent studies have examined short-term memory or memory span for Chinese characters (e.g., Tzeng, Hung, \& Wang, 1977; Yu, Jing, \& Sima, 1984; G. Zhang \& Simon, 1985; W. Zhang, Peng, \& Sima, 1984). In general, the data for Chinese and English stimuli are quite similar, suggesting that basic memorial processes are not language specific. The span for familiar characters and radicals is about 6.5 (G. Zhang \& Simon, 1985; W. Zhang et al., 1984), similar to the span for common English words (e.g., Miller, 1956). As with English stimuli (e.g., Marks \& Jack, 1952; Simon, 1974), there

Correspondence concerning this article may be addressed to either author at the Psychology Department, Box 19528, University of Texas at Arlington, Arlington, TX 76019.

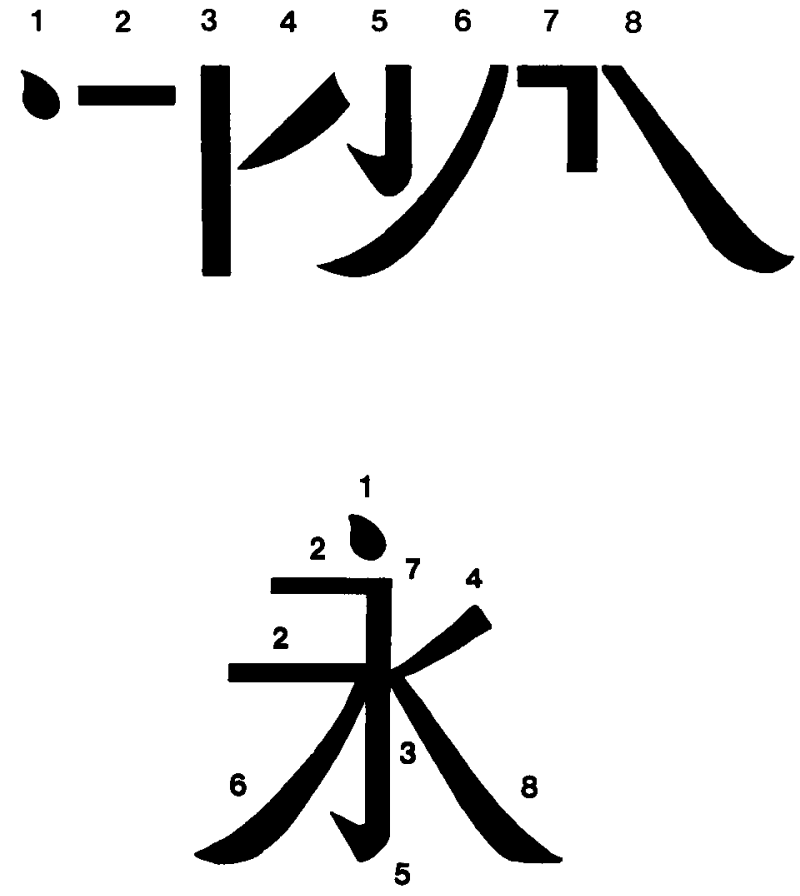

Figure 1. Examples of commonly used strokes used in forming Chinese characters, and a character using these strokes.

is strong evidence for chunking, with memory span being much more constant in units of well-known chunks than in units of radicals or characters. For example, the spans for Chinese characters, two-character words, and common four-character idioms are about $6.6,4.6$, and 3.0 units, respectively (G. Zhang \& Simon, 1985). Finally, as with English stimuli (e.g., Hall, 1954), shortterm memory span for Chinese characters is influenced by linguistic frequency; Yu et al. (1984) reported memory 
spans for high-, medium-, and low-frequency onecharacter words of about $8.3,5.7$, and 3.2 , respectively.

These studies are important for a number of reasons. For one thing, they offer an ecological test for short-term memory models, which have been developed almost exclusively on the basis of words from alphabetic languages. More important for our purposes, Yu et al. (1985) and G. Zhang and Simon (1985) noted that there is evidence for a small nonacoustic short-term memory for Chinese characters whose pronunciations are not known.

In one experiment bearing on the question of visual versus verbal short-term memory, G. Zhang and Simon (1985) studied memory span for lists of homophones, in which every character in the list had exactly the same pronunciation (including "tone," the change in vowel pitch used in Chinese) and found that memory span for these lists was reduced to about 2.8 characters. Since the utility of phonological information is so limited with homophone stimuli, Zhang and Simon argued that nonacoustic encoding was involved for these stimuli, and that the short-term memory capacity for stimuli encoded nonacoustically was severely limited. However, these data are not completely conclusive, for two reasons. First, if subjects recognize the nature of the lists, their recall data may be contaminated by guessing characters with the same pronunciation. Second, homophones necessarily differ in their frequency in the language. Since G. Zhang and Simon also reported that memory span is less for lowthan for high-frequency homophones, the limited memory span for homophonic characters can be interpreted in terms of the limited capacity of a nonacoustic memory, in terms of the ability of subjects to guess characters correctly, or simply as a frequency effect.

In another experiment relevant to the status of a visual short-term memory, G. Zhang and Simon (1985) studied memory span for nonpronounceable radicals, noting that "although educated Chinese can recognize every radical, many radicals do not have commonly used oral names" (p. 194). They found that the span for such radicals was about 2.7 units, very similar to the span for artificially constructed Chinese "characters" (Yu et al., 1984). It is not strictly true that some radicals are not pronounceable, since every radical can be found in dictionaries and has a pronunciation and one or more meanings. It is true that nonpronounceable radicals are used very infrequently as individual characters, and that most people do not know their names. Thus it is not completely clear whether the limited memory span for these radicals reflects the capacity of a visual short-term memory or whether it simply reflects a frequency effect.

In the present study we investigated immediate recall of Chinese characters and radicals and attempted to differentiate contributions from visual and verbal-acoustic short-term memory in several ways. In Experiment 1, we studied immediate recall of individual Chinese radicals. Radicals that differed in orthographic complexity, in frequency as individual characters, and in frequency as components of compound characters were chosen as stimuli, and effects of these variables on memory span were investigated. In Experiments 2, 3, and 4, we studied immediate memory of compound characters that varied widely in frequency and orthographic complexity. In Experiments 3 and 4 , verbal or visual intervening tasks were given prior to list recall, and differential effects of these tasks were examined.

Before presenting the experiments, we note a few relevant aspects of the written Chinese language (Wang, 1973, provides a more detailed summary). Many Chinese characters are formed by combining two or more simple characters. Often part of a compound character, called the phonetic, provides information about the pronunciation of the character, while another, called the signific or radical, provides information about its meaning. However, the meaning of a compound character may or may not be related to the meaning of its radical. Chinese dictionaries are usually arranged by radical, with both radicals and characters that contain a particular radical ordered by number of strokes.

Most Chinese characters and radicals have a distinct meaning and a one-syllable pronunciation; however, many characters are exact homophones. In one popular dictionary, The Far East Chinese Character Dictionary (1985), more than 11,000 characters are listed, but only 1,310 distinctive pronunciations (including tone) are given.

English words and Chinese characters differ in the degree to which pronunciation is related to orthography. The pronunciation of an English word is typically related to the orthographic structure of the word, but this is not true for a Chinese character, unless it has a phonetic component. Part of the reason that it has been difficult to study the role of visual short-term memory using verbal stimuli is the high grapheme-phoneme correspondence for English words. Because of their loose orthographic-phonemic correspondence, Chinese characters are excellent stimuli for studying visual versus acoustic coding in short-term memory.

\section{EXPERIMENT 1}

The main purpose of Experiment 1 was to add to the findings of Yu et al. (1984), Yu et al. (1985), G. Zhang and Simon (1985), and W. Zhang et al. (1984) by investigating immediate memory for Chinese radicals as functions of orthogrpahic complexity, intercharacter frequency, and linguistic frequency.

As noted above, some radicals are used frequently as individual characters, and others are not; the names of the latter characters are not likely to be known even by highly educated Chinese, but can be found in Chinese dictionaries. Radicals from each class were chosen, and are denoted as high frequency (probably pronounceable) and low frequency (probably not pronounceable). Linguistic frequency was determined from norms prepared by the National Institute for Compilation and Translation (1967). These norms rank about 5,000 radicals and characters in 
the Chinese language by frequency. Since literate Chinese are often estimated to know about 5,000 characters ( $\mathrm{Yu}$ et al., 1985), any radical not included in the norms was considered to be of low frequency, and any radical in the norms was considered to be of high frequency for purposes of this study. ${ }^{1}$

In addition, the relative familiarity of radicals was manipulated by choosing radicals that are components of many or few characters in the Far East Dictionary (1985). This familiarity variable is denoted as intercharacter frequency, the number of characters in which a radical appears (in contrast to linguistic frequency, the rank of a radical or character in frequency norms). High and low intercharacter frequency radicals were defined as those that appear in six or more and in fewer than six characters, respectively.

Finally, orthographic complexity of radicals was manipulated. High-complexity radicals were defined as those containing four or more strokes; low-complexity radicals contained three or fewer.

Although linguistic frequency, intercharacter frequency, and complexity are confounded in the language, it was possible to select radicals that allowed these variables to be manipulated reasonably independently. Figure 2 shows sample radicals used.

Of particular interest were the interaction of linguistic frequency with complexity and with intercharacter frequency. For high-frequency radicals, whose pronunciations are presumably well known, small effects of intercharacter frequency and complexity were expected, since prior research suggested that these radicals would act as unified chunks. For low-frequency radicals, whose names are unlikely to be known, effects of intercharacter frequency and complexity were expected. It seemed possible that radicals with low linguistic frequency but high intercharacter frequency could be recalled by using a character-recoding strategy (similar to encoding "DUC" as "DUCK minus K" for an English subject). Because such a strategy would be more effective for radicals that appear in many characters, it was expected that for lowfrequency radicals, memory span would be larger for radicals with high intercharcter frequency. To the extent that a visual short-term memory acts like a "sketch pad" of very limited capacity (Baddeley, 1981, 1986), the memory span for simple low-frequency radicals should be greater than for complex ones, because the number of strokes to remember is less.

\section{Method}

Subjects. The subjects were 10 Chinese graduate students studying at the University of Texas at Arlington. These students, all fluent speakers and readers of Chinese, had received at least 16 years of education and their Bachelor's degrees in Taiwan, where schooling is in Chinese.

Materials. Eight sets of radicals were selected from the Far East Dictionary (1985) and the National Institute (1967) norms by crossing linguistic frequency, intercharacter frequency, and complexity. Each set contained eight radicals. ${ }^{2}$ The dictionary includes a table listing radicals and the number of characters containing each radical, facilitating the selection of high and low intercharacter frequency radicals. The mean number of characters containing the low and high intercharacter frequency radicals used was 2.91 and 20.22, respectively. The mean number of strokes for simple and complex radicals chosen was 2.62 and 6.92 , respectively.

For each of the eight cells in the experiment, eight random lists of radicals were created. Two lists contained two radicals, two contained four, two contained six, and two contained eight. Within each cell, individual radicals were used approximately an equal number of times across lists. Each stimulus list was randomly ordered and hand printed on an index card.

Design and procedure. The subjects were tested individually and attempted to memorize 64 lists, two of each list length for each of the eight list types. The 64 lists were presented in random order at a rate of $500 \mathrm{msec}$ per radical; thus a card containing two radicals was shown for $1 \mathrm{sec}$, a card containing four radicals for $2 \mathrm{sec}$, and so forth. Before the presentation of a list, the experimenter said "ready," then after about $1 \mathrm{sec}$, said "go" and showed the list. The subjects were instructed to try to memorize each list, and were instructed to write down, in any order they wished, as many radicals as they could after list presentation. Recall was in serial order the vast majority of the time, but correct serial order was not considered in the analysis of the data.

\section{Results}

The data were scored in two ways. First, memory span was estimated by the method of G. Zhang and Simon (1985), where memory span for a subject in a given condition is equal to $K+.5 N$. $K$ is the longest list length at which both lists were recalled perfectly, and $N$ is the number of lists longer than $K$ that were recalled perfectly. This provides a relatively crude estimate of memory span, since only list lengths of $2,4,6$, and 8 were used, but is useful in comparing our data with other published data. Second, for each subject, the mean number of radicals recalled at each list length was calculated, providing data comparable to those from Experiments 2, 3, and 4, in which only one list length (8) was used. A Type I error probability of .05 was used for decisions regarding statistical significance in experiments reported here.

Memory span. The mean memory spans for each of the eight list conditions are shown in Table 1 . The mean memory span was considerably higher for high- than for low-frequency radicals $[4.80$ vs. $1.65 ; F(1,9)=295.21$, $M S e=.67]$. The interaction between frequency and intercharacter frequency was significant $[F(1,9)=13.50, M S \mathrm{e}$ $=.53]$. Tests of simple effects showed that for highfrequency radicals the effect of intercharacter frequency was small and nonsignificant, whereas for low-frequency radicals the memory spans for radicals with high and low intercharacter frequency were 2.20 and 1.10 , respectively, a significant difference. The interaction between frequency and complexity was also significant $[F(1,9)=$ $81.00, M S \mathrm{e}=.45]$. Tests of simple effects showed that for high-frequency radicals, the mean spans for simple and for complex radicals were 3.85 and 5.75 , respectively, whereas for low-frequency radicals, the mean spans were 2.05 and 1.25 for simple and complex radicals, respectively; both differences were significant.

Radical recall. The mean number of radicals recalled in each condition at each list length is shown in Table 1. 
RADICALS

\begin{tabular}{|c|c|c|c|c|c|}
\hline Example & Pronounciation & Translation & $\begin{array}{l}\text { Linguistic } \\
\text { Frequency }\end{array}$ & Complexity & $\begin{array}{c}\text { Inter-character } \\
\text { Frequency }\end{array}$ \\
\hline 寸 & Tsuenn & Inch & High & 3 & High \\
\hline 干 & Gan & Shield & High & 3 & Low \\
\hline 狇 & Yeu & Feather & High & 6 & High \\
\hline Fri & Chern & Minister & High & 6 & Low \\
\hline ン & Bing & Ice & Low & 2 & High \\
\hline$\sim$ & Mih & Cap & Low & 2 & Low \\
\hline 采 & Jyh & Worm & Low & 7 & High \\
\hline ye & Bo & Limp & Low & 5 & Low \\
\hline
\end{tabular}

CHARACTERS

\begin{tabular}{|c|c|c|c|c|}
\hline Example & Pronounciation & Translation & Linguistic Frequency & Complexity \\
\hline$\pi$ & Yuan & Beginning & High & 4 \\
\hline 游 & Sheng & Sound & High & 17 \\
\hline 仆 & $\mathrm{Pu}$ & Fall & Medium & 4 \\
\hline 㳡迷 & Mhi & Squint & Medium & 15 \\
\hline 无 & Wu & Nothing & Low & 4 \\
\hline 第 & Li & Dragon's Saliva & Low & 15 \\
\hline
\end{tabular}

Figure 2. Examples of radicals and characters used, with some of their characteristics.

The main effect of frequency $[F(1,9)=476.93, M S e=$ .71] and the interactions between frequency and complexity $[F(1,9)=51.01, M S \mathrm{e}=.74]$ and between frequency and intercharacter frequency $[F(1,9)=12.18, M S \mathrm{e}=$ .62] were significant; the nature of these effects is the same as for memory span. The effects involving list length are of no particular interest, and add little to the data pattern summarized above.

Note that the mean number of radicals recalled at list lengths of 6 and 8 was almost always higher than the memory span estimates. It is our opinion that item recall provides a more sensitive measure than memory span given the procedures used; therefore, item recall was used as the dependent variable in Experiments 2,3, and 4, with list length held constant at 8 .

Intrusion errors. Errors were categorized as follows: Acoustic/phonological errors were homophones of or shared at least a vowel and a consonant with a nonrecalled item from the study list. Visual errors were responses that shared several contiguous orthographic features (strokes 
Table 1

Memory Span and Immediate Recall for Chinese Radicals

\begin{tabular}{|c|c|c|c|c|c|}
\hline \multirow{2}{*}{$\begin{array}{l}\text { Intercharacter } \\
\text { Frequency }\end{array}$} & \multirow{2}{*}{$\begin{array}{l}\text { List } \\
\text { Length }\end{array}$} & \multicolumn{2}{|c|}{ High Frequency } & \multicolumn{2}{|c|}{ Low Frequency } \\
\hline & & Simple & Complex & Simple & $\mathrm{C}$ \\
\hline
\end{tabular}

Memory Span for Radicals as a Function of Linguistic Frequency, Intercharacter Frequency, and Complexity (Experiment 1)

$\begin{array}{llllll}\text { High } & \mathrm{n} / \mathrm{a} & 3.90 & 5.60 & 2.40 & 2.00 \\ \text { Low } & \mathrm{n} / \mathrm{a} & 3.80 & 5.90 & 1.70 & 0.50\end{array}$

Number of Radicals Recalled as a Function of Linguistic Frequency, Intercharacter Frequency, Complexity, and List Length

\begin{tabular}{llllll} 
High & 2 & 2.00 & 2.00 & 1.95 & 1.95 \\
& 4 & 3.50 & 3.95 & 2.90 & 1.90 \\
& 6 & 5.00 & 5.55 & 3.15 & 1.80 \\
\multirow{4}{*}{ Low } & 8 & 5.20 & 5.40 & 2.60 & 2.30 \\
& 2 & 1.95 & 2.00 & 1.80 & 1.15 \\
& 4 & 3.50 & 3.90 & 1.80 & 1.05 \\
& 6 & 4.80 & 5.85 & 2.15 & 1.25 \\
& 8 & 3.85 & 6.50 & 2.45 & 1.75 \\
\hline
\end{tabular}

Note $-\mathbf{n} / \mathbf{a}=$ not applicable.

or substructures) with a list item. In Experiment 1 every radical appeared on several recall lists, and many errors were radicals that had been presented on other trials; these were classified as other-stimulus errors. This kind of error did not occur in the other experiments. Errors that could not be assigned to one of these categories were called miscellaneous errors. These were usually radicals or characters in the language that were not related to stimuli shown, although one or two semantic errors (synonyms of list items) occurred in each experiment and were also classified as miscellaneous. The number of errors of each type is shown in Table $2 .^{3}$

Many errors were radicals presented on other trials. When a high-frequency list was presented, $84 \%$ of errors were other high-frequency radicals; when a low frequency list was presented, $52 \%$ of errors were other lowfrequency radicals. Other-stimulus errors probably represent educated guesses, biased in favor of highfrequency radicals, and are not of particular interest here.

When the list contained high-frequency radicals, there were several acoustic errors. These errors never occurred on low-frequency lists, where errors tended to involve responses that were visually similar to a list item. Although there were also several visual errors on highfrequency, low-complexity lists, these errors differed from visual errors on low-frequency lists. Visual errors on highfrequency, low-complexity lists tended to be other radicals or characters with one more or one less stroke than a presented item, suggesting misperception of an item.
Visual errors on low-frequency lists tended to be partial radicals (a few strokes from a presented radical), suggesting that incomplete, rather than incorrect, perception or storage of an item is common for low-frequency stimuli.

\section{Discussion}

For high-frequency radicals, those used frequently as individual characters in the language, memory span was relatively high and was not significantly influenced by intercharacter frequency. However, there was an unexpected effect of complexity for high-frequency radicals, namely better recall of complex radicals. One possible explanation of this effect is that complex radicals are more distinctive. Ovid Tzeng (personal communication) noted, "As the number of strokes within a character increases, there begins [sic] to emerge distinctive graphemic patterns which may serve as useful retrieval cues in addition to the phonological codes." This interpretation is supported by the error data, which show that for high-frequency radicals, there were more than twice as many intrusion errors on simple as on complex lists, and that many of these indicated visual confusions with other radicals. To the degree that high-complexity radicals are distinctive, or contain redundant components, a quick glance will be more likely to result in accurate perception, leading to improved recall scores.

The data from low-frequency radicals, those that do not appear frequently as individual characters in the language, are quite interesting and are of theoretical importance. Memory for low-frequency radicals was very limited and was strongly influenced by intercharacter frequency and complexity. On the average, our Chinese subjects could recall less than one and a half low intercharacter frequency, complex, low-frequency radicals. If the radical was either simple or of high intercharacter frequency, recall increased to about two radicals, and if it was both simple and of high intercharacter frequency, recall increased to about two and a half radicals.

That low-frequency radicals that appear in many compound characters are recalled better than those that do not may reflect differential utility of various mnemonic strategies. For example, subjects may try to associate an unknown radical with a known character in which it appears, and store that character verbally for later recall, much as English subjects may use a word-plustransformation mnemonic to recall a nonsense syllable. A strategy of this type would clearly be more effective for radicals appearing in many compound characters. For low-frequency radicals, the recall difference between high

Table 2

Number of Intrusion Errors of Different Types as a Function of Radical Complexity and Frequency (Experiment 1)

\begin{tabular}{ccrccc}
\hline & \multicolumn{5}{c}{ Radical Complexity and Frequency (Experiment 1) } \\
\cline { 3 - 6 } Frequency & Complexity & Visual & Acoustic & Other Stimuli & Miscellaneous \\
\hline High & High & 3 & 6 & 12 & 12 \\
High & Low & 23 & 5 & 25 & 22 \\
Low & High & 111 & 0 & 42 & 30 \\
Low & Low & 58 & 0 & 53 & 19 \\
\hline
\end{tabular}


and low intercharacter frequency radicals may be analogous to effects of meaningfulness on recall of nonsense syllables by English subjects.

The very limited recall scores for low-frequency, low intercharacter frequency, complex radicals may represent the limited capacity of a visual short-term memory. The complexity difference for radicals with low linguistic and intercharacter frequency may indicate that, for these stimuli, the capacity of a visual short-term memory is measurable in terms of number of strokes or chunks of strokes.

\section{EXPERIMENT 2}

The data from Experiment 1 suggest that both verbal and visual characteristics of stimuli are involved in shortterm memory for Chinese radicals. Visual characteristics, such as complexity, seem to be important when radicals are of low linguistic and intercharacter frequency. If so, similar interactions of frequency and complexity should be obtained for compound Chinese characters. In addition, since there are many times more characters than radicals in the language, the use of characters allows more precise matching of frequencies for stimuli of different types.

In Experiment 2, Chinese characters varying in frequency and in complexity were presented for immediate free recall. Lists of eight items were used in all conditions. High- and medium-frequency characters were chosen from the National Institute (1967) norms, with high-frequency characters chosen from the 300 most frequent characters in the norms and medium-frequency characters chosen from characters occupying ranks above 3,000 . If estimates that literate Chinese know about 5,000 characters are reasonably accurate, high- and mediumfrequency characters are likely to be known by our subjects. Characters from the Far East Dictionary (1985) that did not appear in the frequency norms were chosen as a low-frequency set. We assumed that our subjects were unlikely to know these characters or their pronunciations. These assumptions were examined by testing whether subjects could pronounce the medium- and low-frequency characters used; there was no doubt that the highfrequency characters were very well known.

\section{Method}

Subjects. The subjects were 11 Chinese graduate students from the same population as in Experiment 1

Materials. Six sets of characters were selected by crossing frequency (high, medium, or low) and complexity (high or low). Simple characters contained 5 or fewer strokes $(M=3.40)$, whereas complex characters contained 10 or more strokes $(M=12.98)$. The mean frequency ranks were 152.7 and 149.3 for high-frequency complex and simple characters, respectively, and were 3689.5 and 3692.5 for medium-frequency complex and simple characters, respectively. Low-frequency characters did not appear in the $\mathrm{Na}$ tional Institute (1967) frequency norms; care was taken not to select low-frequency characters that contained a well-known phonetic. Each character set contained 16 characters and was randomly divided into two 8-item lists. In addition, four lists, each containing $8 \mathrm{com}$ mon characters, were selected for practice lists. Examples of characters used are shown in Figure 2.

A pronunciation test was developed for the medium- and lowfrequency items. For each character, four pronunciation options, one of which was correct, were written in Mandarin phonetic symbols that are taught in elementary schools in Taiwan, and are used as a major pronunciation guide in every Chinese dictionary.

Design and procedure. The subjects were tested individually, as in Experiment 1 . After the four practice lists were administered, the 12 stimulus lists were presented in random order. Each eightitem list was hand printed in random order on an index card, and was presented for a 4-sec study period (the same $500 \mathrm{msec}$ per item rate as in Experiment 1). The subjects were given as much time as they desired for immediate written free recall of each list. After the 12 recall trials, the pronunciation test was administered, and the subjects were encouraged to guess if they did not know the correct answer for an item.

\section{Results}

Character recall. The mean number of characters recalled in each condition is given in Table 3 . The main effect of frequency was significant $[F(2,10)=163.41$, $M S e=.70]$, with high-frequency items being easiest to recall and low-frequency items the most difficult; all pairwise differences among frequency levels were significant. In addition, the interaction between frequency and complexity was significant $[F(2,10)=13.98, M S \mathrm{e}=.39]$. As Table 3 shows, for high- and medium-frequency characters, effects of complexity were quite small, whereas for low-frequency characters, orthographically simple characters were much easier to recall than complex ones; this simple effect was significant.

Intrusion errors. On lists of high-frequency characters, there were 11 acoustic and 7 miscellaneous errors, and on lists of medium-frequency characters, there were 15 acoustic, 1 visual, and 8 miscellaneous errors; all were other common characters in the language. On lists of lowfrequency characters, there were 23 visual and 7 miscellaneous errors. Forty-eight percent of the low-frequency visual errors were common pronounceable characters, visually similar to a presented item; the others were noncharacter stroke patterns. As in Experiment 1, errors in dicating partial storage or recall of visual information occurred only on low-frequency lists.

Pronunciation test. Our subjects knew the correct pronunciations of most of the medium-frequency characters $(97 \%)$ but not of most of the low-frequency characters $(34 \%)$. The correct pronunciation rate for lowfrequency characters was not significantly above chance (25\%).

Table 3

Immediate Recall of Eight-Character Lists as a Function of Frequency and Complexity (Experiment 2)

\begin{tabular}{cccc} 
Complexity & $\begin{array}{c}\text { High } \\
\text { Frequency }\end{array}$ & $\begin{array}{c}\text { Medium } \\
\text { Frequency }\end{array}$ & $\begin{array}{c}\text { Low } \\
\text { Frequency }\end{array}$ \\
\hline Simple & 6.50 & 3.64 & 2.73 \\
Complex & 6.36 & 3.82 & 1.05 \\
\hline
\end{tabular}




\section{Discussion}

The character recall data show essentially the same effects of frequency and complexity as the radical recall data from Experiment 1. Recall declined sharply as characters became less frequent. Unlike high- or mediumfrequency characters, low-frequency characters seem to be stored in visual form in short-term memory. For lowfrequency characters, recall was strongly influenced by complexity, and intrusion errors indicated visual and not acoustic confusions, and often indicated partial storage or recall of visual information.

The data suggest that both high- and medium-frequency characters are maintained (with different degrees of difficulty) in verbal form in short-term memory. There were no effects of orthographic complexity for these characters, and the intrusion data show almost no indication of visual confusions, only acoustic confusions.

\section{EXPERIMENT 3}

The results of Experiment 2 suggest that pronounceable characters are stored in short-term memory in verbal form, whereas characters whose pronunciations are not known are stored visually. Experiment 3 was designed to provide converging evidence for this interpretation. In this experiment, the subjects were given the lists from Experiment 2 to be recalled, but before recall an intervening task that required either a verbal-acoustic or a visual discrimination was administered.

It was expected that the verbal-acoustic, but not the visual, intervening task would interfere with recall for high- and medium-frequency lists and that recall of lowfrequency items would show interference from the visual, but not from the verbal-acoustic, task. Such differential interference has been found in experiments using English words and pictures (e.g., Brooks, 1968; den Heyer \& Barrett, 1971).

\section{Method}

Subjects. The subjects were 10 Chinese graduate students from the same pool used in the other experiments.

Stimuli. The stimuli used in Experiment 2 were also used in this experiment.
In the verbal-acoustic intervening task, the subjects were shown a Chinese character followed by four other characters, and were asked to select the character that was an exact homophone of the first one. In the visual intervening task, the subjects were shown a random pattern of five dots (from a $5 \times 5$ matrix) followed by four other random-dot patterns, and were asked to select the pattern that was identical to the first one. Thirty-two problems of each type were handwritten or drawn on index cards. For a given subject, eight four-problem sets of verbal problems, and eight four-problem sets of visual problems were randomly selected.

Design and procedure. The design and procedure were similar to those of Experiment 2. The subjects were run individually, and they recalled 4 practice and 12 experimental lists. Before recalling any list, the subjects were given one of the sets of four intervening problems. Two of the practice lists involved the visual and two the verbal intervening task.

During the experimental recall trials, the type of intervening task was crossed with the other variables, so that, within each of the six combinations of frequency and complexity, a subject received one list with the visual and one list with the verbal intervening task. For each subject, lists and intervening tasks were randomly ordered within these restrictions.

\section{Results}

Character recall. The recall data from Experiment 3 are shown in Table 4. A significant main effect of frequency was found $[F(2,18)=50.94, M S e=3.47]$. The mean numbers of high-, medium-, and low-frequency characters recalled were $5.70,2.70$, and 1.65 , respectively; all pairwise differences were significant. As in the earlier experiments, the interaction between frequency and orthographic complexity was significant $[F(2,18)=8.49$, $M S e=1.07]$. The pattern of the interaction was the same as in Experiment 1, with small, but significant, complexity differences for high- and medium-frequency characters such that more complex than simple characters were recalled. For low-frequency characters, as in Experiments 1 and 2, over twice as many simple as complex characters were recalled.

More important, the interaction between frequency and intervening task was significant $[F(2,18)=6.76, M S \mathrm{e}$ $=1.24]$, and the pattern of the interaction was as expected. For high- and medium-frequency characters, the verbal task interfered with recall significantly more than did the visual task $[F(1,18)=18.58, M S e=1.55$, in a supplementary analysis of variance]. Compared with the

Table 4

Immediate Recall for Chinese Characters as a Function of Frequency, Complexity, and Intervening Task

\begin{tabular}{|c|c|c|c|c|c|c|}
\hline \multirow{2}{*}{$\begin{array}{l}\text { Intervening } \\
\text { Task }\end{array}$} & \multicolumn{2}{|c|}{ High Frequency } & \multicolumn{2}{|c|}{ Medium Frequency } & \multicolumn{2}{|c|}{ Low Frequency } \\
\hline & Simple & Complex & Simple & Complex & Simple & Complex \\
\hline \multicolumn{7}{|c|}{$\begin{array}{l}\text { Immediate Recall of Eight-Item Lists Presented at a Rate of } \\
500 \mathrm{msec} \text { per Character (Experiment 3) }\end{array}$} \\
\hline $\begin{array}{l}\text { Visual } \\
\text { Verbal }\end{array}$ & $\begin{array}{l}5.90 \\
5.10\end{array}$ & $\begin{array}{l}6.40 \\
5.40\end{array}$ & $\begin{array}{l}3.50 \\
1.40\end{array}$ & $\begin{array}{l}3.40 \\
2.50\end{array}$ & $\begin{array}{l}2.10 \\
2.40\end{array}$ & $\begin{array}{l}0.90 \\
1.30\end{array}$ \\
\hline \multicolumn{7}{|c|}{$\begin{array}{l}\text { Immediate Recall of Eight-Item Lists Presented at a Rate } \\
\text { of } 1,000 \text { msec per Character (Experiment 4) }\end{array}$} \\
\hline $\begin{array}{l}\text { Visual } \\
\text { Verbal }\end{array}$ & $\begin{array}{l}6.93 \\
6.93\end{array}$ & $\begin{array}{l}7.43 \\
7.00\end{array}$ & $\begin{array}{l}4.29 \\
3.79\end{array}$ & $\begin{array}{l}4.00 \\
3.57\end{array}$ & $\begin{array}{l}2.71 \\
3.86\end{array}$ & $\begin{array}{l}1.14 \\
2.14\end{array}$ \\
\hline
\end{tabular}


data from Experiment 2, the visual intervening task decreased character recall for high- and medium-frequency characters about half a character, whereas the verbal intervening task decreased it by an average of 1.5 characters. Although the data were ordered as expected, the simple effect of intervening task was not significant for low-frequency characters, however. Compared with Experiment 2 , the verbal intervening task did not decrease recall for low-frequency characters whereas the visual intervening task decreased it by .4 characters.

Intrusion errors. The intrusion data were very similar to those in Experiments 1 and 2, and did not vary much as a function of the intervening task. On high- and medium-frequency lists, there were 62 acoustic, 8 visual, and 22 miscellaneous errors; all were other pronounceable characters. Six of the visual errors were made on low-complexity lists, a pattern also found in Experiment 1 . On low-frequency lists there were 29 visual, 1 acoustic, and 9 miscellaneous errors. Fifty-two percent of the visual errors were other characters in the language; the rest were partial characters.

\section{Discussion}

Experiment 3 replicated the frequency and complexity effects found in previous experiments. Short-term memory for high- and medium-frequency characters was influenced by frequency; weakly influenced by orthographic complexity, with slightly better recall for complex characters; and interfered with more by a verbal than by a visual intervening task. The differential effects of the two intervening tasks provide support for the interpretation that pronounceable characters are maintained in verbal form in short-term memory. Short-term memory for lowfrequency characters was quite limited and was influenced by orthographic complexity, with better recall of simple than of complex characters. The tendency for interference from a visual, but not a verbal, intervening task and the pattern of intrusion errors provide partial support for the conclusion that immediate recall for these characters reflects a limited visual short-term memory.

\section{EXPERIMENT 4}

It seemed likely that limited recall of low-frequency characters, suggesting a floor effect, resulted in the failure to find a significant difference between the two intervening tasks for these items in Experiment 3. The purpose of Experiment 4 was to increase recall for low-frequency characters in order to provide a more sensitive test of differential interference effects. Experiment 4 was identical to Experiment 3, except that the subjects were given more time to study each list prior to recall.

\footnotetext{
Method

Subjects. The subjects were 14 students from the same pool as in the other experiments.

Stimuli, design, and procedure. The subjects were given $8 \mathrm{sec}$ to study each list (1,000 msec per item) as opposed to the $500 \mathrm{msec}$ per item used in prior experiments.
}

\section{Results}

Character recall. The mean number of characters recalled in each condition is given in Table 4. Comparison of the data from Experiments 3 and 4 shows that the added study time increased recall by an average of 1.1 characters. As in the previous studies, the main effect of frequency was significant $[F(2,26)=177.12, M S e=2.45]$, with the order of conditions as before. The main effect of complexity $[F(1,13)=12.18, M S e=.99]$ and the interaction between complexity and frequency $[F(2,26)$ $=5.95, M S e=2.33]$ were also significant. For highand medium-frequency characters, there were small and nonsignificant effects of complexity, but for lowfrequency characters, about twice as many simple as complex characters were recalled, as in the prior experiments.

The interaction between frequency and intervening task was significant $[F(2,26)=6.51, M S e=1.46]$. Table 4 shows that recall of high-frequency words was essentially at the ceiling, and there was no differential effect of task for these items. For medium-frequency characters, recall was better $(p<.06)$ when the intervening task was visual, whereas for low-frequency characters, recall was significantly better when the intervening task was acoustic.

Intrusion errors. On high- and medium-frequency lists, there were 49 acoustic, 2 visual, and 12 miscellaneous errors; all were other characters in the language. On lowfrequency lists there were 21 visual and 1 miscellaneous errors; $52 \%$ of the visual errors were other characters in the language, and the remainder were partial characters.

\section{Discussion}

Giving subjects more time to study the lists produced better recall, moving recall for low-frequency characters off the floor (and moving recall for high-frequency characters to the ceiling). This allowed the effects of the intervening task to become apparent for low-frequency characters, and for these stimuli there was more interference from a visual than a verbal intervening task. The combination of data from Experiments 3 and 4 provides strong support for dual-code short-term storage, with higher frequency characters maintained in verbal form while lowfrequency characters are maintained in visual form. Under appropriate conditions, a verbal intervening task interferes more with short-term memory for pronounceable characters and a visual intervening task interferes more with short-term memory for characters whose pronunciation is not known.

\section{GENERAL DISCUSSION}

Many studies in the 1960s supported the idea that the primary coding of verbal material in short-term memory is phonological (e.g., Conrad, 1964; Kintsch \& Buschke, 1969), although access to other codes is certainly possible (e.g., Shulman, 1970). Thus, research reported by Rozin, Poritsky, and Sotsky (1971), which showed that American children with serious reading problems could learn to read Chinese characters, and clinical research on 
aphasics by Sasanuma (e.g., 1975; Sasanuma \& Fujimura, 1971), suggesting differential processing of Japanese Kanji (logographic) and Kana (syllabic) material, was of great interest. This research suggested that processing of logographic and alphabetic materials may entail different processes, such that in reading Chinese the phonological recoding stage commonly found in alphabetic text would be bypassed and semantic content would be addressed relatively directly.

However, later research (e.g., Hung \& Tzeng, 1981; Tzeng et al., 1977) strongly suggested that processing of Chinese and other nonalphabetic languages by fluent readers of the language involves phonological coding. Since then, Tzeng and Wang (1983) provided evidence that both acoustic and visual memory are involved in processing of logographs. The present research does not rule out the possibility of dual storage for high-frequency Chinese characters, but does indicate a priority for verbal codes. For characters whose pronunciation is known, short-term memory capacity is very similar to that for English, and this capacity is not influenced by orthographic complexity or, for radicals, intercharacter frequency. Short-term memory for frequent characters is interfered with more by a verbal than a visual intervening task. Intrusion errors tend to involve acoustic confusions; the few visual errors that do occur tend to involve less visually distinctive low-complexity stimuli. For high- and mediumfrequency characters and radicals, the combination of visual confusion errors on low-complexity lists, found primarily in Experiments 1 and 3, and the better recall on high-complexity lists, found in the same experiments, is of some interest. This pattern suggests that visual distinctiveness among characters or radicals is important, particularly under conditions in which unrelated characters are briefly presented.

For present purposes, the most important finding is that low-frequency Chinese radicals and characters, whose pronunciations and meanings are not apt to be known, seem to be stored in visual form in short-term memory. Unlike English, in which an orthographically legal unknown word or nonword is likely to be pronounceable, for a Chinese reader an unknown character is little more than a "character-like" visual scramble if the character does not have a familiar signific or phonetic component. It may be recognizable as "legal," but there is no way to give it a verbal code without the help of a dictionary.

Memory for such characters is very limited and is influenced by orthographic complexity and, for radicals, intercharacter frequency. Recall for these characters is interfered with more by a visual than a verbal intervening task; overt recall errors show visual, not acoustic, confusions and these visual errors are often partial characters or radicals. In summary, for Chinese subjects, the Chinese language provides some stimuli that seem to be stored in verbal or acoustic form and other stimuli, uncommon characters and radicals, that apparently are stored visually in short-term memory.

These data support the concept of a visual short-term memory of very limited capacity (e.g., Baddeley et al.,
1975; Phillips, 1974; Phillips \& Christie, 1977; Yu et al., 1984). G. Zhang and Simon (1985) suggested that the visual short-term memory capacity for Chinese characters is about two or three characters, but our research suggests that it is even less, often being one or two characters. In fact, the basic unit for visual short-term memory of Chinese characters may be better defined in terms of strokes, or chunks of strokes, than in terms of characters.

The apparent existence of some Chinese characters whose initial storage is verbal-acoustic and others whose initial storage is visual makes Chinese characters very interesting for research. Some research questions involve basic questions of how Chinese deal with these materials, for example, when looking up an unknown character in a dictionary. The importance of character distinctiveness and the role of dual codes in the reading process, where characters are presented in context, is also well worth investigating.

Other research may involve taking advantage of differences among characters in attacking theoretical questions. For example, findings such as the modality effect (e.g., Crowder \& Morton, 1969), the recall advantage in the recency portion of the serial-position curve for material that has been heard rather than read, are often interpreted in terms of mandatory verbal coding of visually presented material. Tzeng and Wang (1983) showed that there is a modality effect for Chinese characters, which, unlike English visual presentation, produces better recall at the nonrecency positions in the serial-position curve.

It would be of some interest to add to this research, by using Chinese characters of varying frequency and examining how serial-position curves vary as a function of type of character and mode of presentation. One would expect that low-frequency characters would yield no modality effect or a reverse modality effect if low-frequency characters are stored visually. The existence of many homophones of most Chinese characters adds another interesting facet to such studies.

Finally, although questions of processes involved in visual short-term memory were not addressed by the research presented here, Chinese characters seem promising as stimuli to answer such questions. Chinese characters provide more control over nonlinguistic variables than do pictures, and more control over storage codes than do English materials, making them very useful as stimulus materials.

\section{REFERENCES}

BADDELEY, A. D. (1981). The concept of working memory: A view of its current state and probable future development. Cognition, 10, 17-23.

Baddeley, A. D. (1986). Working memory. Oxford: Clarendon Press.

Baddeley, A. D., Grant, S., Wight, E., \& Thomson, N. (1975). Imagery and visual working memory. In P. M. A. Rabbitt \& S. Dornic (Eds.), Attention and performance V (pp. 205-217). London: Academic Press.

BADDELeY, A. D. , LneBERMAN, K. (1980). Spatial working memory. In R. S. Nickerson (Ed.), Attention and performance VIII (pp. 521539). Hillsdale, NJ: Erlbaum. 
Brooks, L. R. (1968). Spatial and verbal components of the act of recall. Quarterly Journal of Experimental Psychology, 10, 349-368.

Cermak, G. W. (1971). Short-term recognition memory for complex free-form figures. Psychonomic Science, 25, 209-211.

ConRad, R. (1964). Acoustic confusion in immediate memory. British Journal of Psychology, 55, 75-84.

Crowder, R. G., Morton, J. (1969). Precategorical acoustic storage (PAS). Perception \& Psychophysics, 5, 365-373.

DEN HEYER, K., \& BARRETT, B. (1971). Selective loss of visual and verbal information in STM by means of visual and verbal interpolated tasks. Psychonomic Science, 25, 100-102.

Far East Chinese character dictionary. (1985). Taipei, Taiwan: Far East Frick, R. (1985). Testing visual short-term memory: Simultaneous versus sequential presentations. Memory \& Cognition, 13, 346-356

HALL, J. F. (1954). Learning as a function of word frequency. American Joumal of Psychology, 67, 138-140.

HUNG, D. L., \& TzENG, O. J. L. (1981). Orthographic variations and visual information processing. Psychological Bulletin, 90, 377-414.

KINTSCH, W., \& BUSCHKE, H. (1969). Homophones and synonyms in short-term memory. Joumal of Experimental Psychology, 80, 403-407.

Laughery, K. R., Welte, J. W., \& Spector, A. (1973). Acoustic and visual coding in primary and secondary memory. Journal of $E x$ perimental Psychology, 99, 323-329.

MARKS, M. R., \& JACK, O. (1952). Verbal context and memory span for meaningful material. American Journal of Psychology, 65, 298-300.

Miller, G. A. (1956). The magical number seven plus or minus two: Some limits on our capacity for processing information. Psychological Review, 63, 81-97.

National Institute for Compilation and Translation. (1967). A study on the high frequency words used in Chinese elementary school reading materials. Taipei, Taiwan: Chung Hwa.

PHILLIPS, W. A. (1974). On the distinction between sensory storage and short-term visual memory. Perception \& Psychophysics, 16, 283-290.

Philuips, W. A., \& Christie, D. F. M. (1977). Interference with visualization. Quarterly Journal of Experimental Psychology, 29, 639-650.

RozIN, P., Porrsky, S., \& SotsKy, R. (1971). American children with reading problems can easily learn to read English represented by Chinese characters. Science, 171, 1264-1267.

Sasanuma, S. (1975). Kana and Kanji processing in Japanese aphasics. Brain \& Language, 2, 141-157.

Sasanuma, S., \& Fujimura, O. (1971). Selective impairment of phonetic and nonphonetic transcription of words in Japanese aphasic patients: Kana vs. Kanji in visual recognition and writing. Cortex, 7, 1-18.

Shulman, H. G. (1970). Encoding and retention of semantic and phonemic information in short-term memory. Journal of Verbal Learning \& Verbal Behavior, 9, 499-508.

Simon, H. A. (1974). How big is a chunk? Science, 183, 482-488.

Tzeng, O. J. L., Hung, D. L., \& WANG, W. S.-Y. (1977). Speech recoding in reading Chinese characters. Joumal of Experimental Psychology: Human Learning \& Memory, 3, 621-630.
Tzeng, O. J. L., \& Wang, W. S.-Y. (1983). The first two R's. American Scientist, 71, 238-243.

WANG, W. S.-Y. (1973). The Chinese language. Scientific American, 228, $51-60$.

Yu, B., JiNG, Q., \& SiMA, H. (1984). STM capacity for Chinese words and phrases under simultaneous presentation. In National Academy of Sciences, Chinese Academy of Sciences, Issues in cognition: Proceedings of a joint conference in psychology (pp. 317-328). Washington: National Academy of Science and American Psychological Association.

Yu, B., Zhang, W., Jing, Q., Peng, R., Zhang, G., \&imon, H. A. (1985). STM capacity for Chinese and English language materials. Memory \& Cognition, 13, 202-207.

ZhanG, G., \& Simon, H. A. (1985). STM capacity for Chinese words and idioms: Chunking and acoustical loop hypotheses. Memory \& Cognition, 13, 193-201.

Zhang, W., Peng, R., \& Sima, H. (1984). STM capacity for Chinese words and idioms with visual and auditory presentations. In National Academy of Sciences, Chinese Academy of Sciences, Issues in cognition: Proceedings of a joint conference in psychology (pp. 331-344). Washington: National Academy of Science and American Psychological Association.

\section{NOTES}

1. Radicals denoted as low frequency correspond in frequency to the low-frequency characters used in Experiments 2, 3, and 4, whereas radicals denoted as high frequency have a mean frequency rank (about 1,020 ) between those of the high- and medium-frequency characters used in the other experiments. The fact that there are many more characters than radicals in the language made it impossible to match radical and character frequency precisely across studies. (There are only 216 radicals included among the approximately 11,000 entries in the Far East Chinese Character Dictionary, 1985.)

2. Only 13 low-complexity radicals are listed in the frequency norms, 7 of high and 6 of low interchararacter frequency. Three radicals of appropriate intercharacter frequency whose pronunciation can be assumed to be known because they are also Mandarin phonetic symbols (see Experiment 2, Method section) were included as high-frequency radicals. Because the primary variable of interest was not frequency per se, but whether or not the pronunciation of an item was apt to be known, this seemed justifiable.

3. Only descriptive data are presented. Chi-square tests were run, and in every experiment the test indicated that the error patterns at different levels of frequency were significantly different. But since the assumption of independent frequencies within cells was not met, because many subjects contributed one or more errors to several cells, the chi-square tests cannot be interpreted cleanly.

(Manuscript received February 23, 1987; revision accepted for publication November 23, 1987.) 